apparently largely spread-out attachment to the uterine surface doubtless accounted for the edge not being felt by the passage through the os of two phalanges of my finger. The woman was not blanched or exhausted to the extent which might have been expected and had borne the whole of the most trying operation bravely, which was much more quickly performed than might appear from its description in writing.

In this case a departure was made by me from the instructions of text-books - to rupture the membranes at the edge of the placenta and to perform version by grasping the first foot met with. But the placenta was one of the very largest $I$ have ever seen and there was my previous experience of her confinements to guide me-namely, that there was no pelvic deformity or flattening, the os was usually quickly dilated, an unrepaired perineum existed, and that the patient possessed great courage and self-control-hence I preferred to perforate the placenta and, finding the head low down in the pelvis with firm uterine contractions, extracting rapidly with forceps to attempting podalic version.

Recovery was uneventful, the temperature and lochia were normal, and all symptoms of anæmia quickly disappeared with good food and nursing.

Husbands Bosworth, Rugby.

\section{NOTE ON "RELAPSES" IN SCARLET FEVER.}

BY A. BANKIER SLOAN, M.D. GLASG.,

ASSISTANT MEDICAL OFFICER, METROPOLITAN ASYLUMS BOARD FEVER HOSPITALS, AND LATE ASSISTANT MEDICAL OFFICER TO THE RUCHILI, FEVER HOSPITAL, GLASGOW.

MY attention was attracted by a note in THE LANCET of Nov. 29th, 1902 , p. 1458 , on a Case of Scarlet Fever followed by an interesting Relapse, by Dr. Henry Fraser. On looking up the annual report of the Metropolitan Asylums Board for 1901 I find that "relapses" occurred in 154 out of 14,143 cases of scarlet fever, or in 1.09 per cent. That "relapses" do occur, I think, is admitted by practically all competent authorities, and I scarcely think that Dr. Fraser will find "many" men who have seen much hospital work deny the fact. Personally, from my own limited experience I have no doubt that they do occur. As it happens I have at the present time two typical cases under my care.

$\mathrm{C}_{\mathrm{ASE}}$ 1.-A girl, aged five years, was admitted into the Western Fever Hospital on Oct. 7th, 1902. She had sickened on the 6th and the rash had appeared on the following day. When admitted the tongue was moist, clean, and red and showing prominent papillæ; the mucous membrane of the palate and fauces was injected and the tonsils showed pultaceous exudate; there was a typical, general, punctate erythema; the temperature was $99^{\circ} \mathbf{F}$. On the 10th right otorrhoe $\mathrm{began}$ and on the 20th it was noted that she was desquamating typically. On Nov. 7th, after having been out of bed for some days, she developed a general, bright, punctate erythema; her temperature was $99^{\circ} 6^{\circ}$; her throat was distinctly injected and her tongue was red. On Dec. 4th (being the sixtieth day of the disease) I specially examined her and found that she was going through a second typical general desquamation.

CASE 2. - A boy, aged four years, was admitted into the Western Fever Hospital on Nov. 4th, 1902. The history was that four weeks previously to admission he had suffered from vomiting and diarrhoea, and that from that date up to the time of admission he had had rhinorrhoea. On admission he was desquamating freely on the hands; he had excoriated nostrils and a profuse rhinorrhcea ; the throat was slightly congested. He was well and had been out of bed for some days when on Dec. 2nd his temperature rose; he developed a bright, general, punctate erythema, the throat became swollen and injected, and the glands at the angle of the jaw were tender and enlarged. The maximum temperature was $102.4^{\circ} \mathrm{F}$. The purulent rhinorchoea had recurred and his throat was now rather "dirty."

Both these cases I consider undoubted relapses. I have also under my care another case which I look upon as a "relapse," but as the original desquamation was not profuse I do not detail it. I do not care for the term "relapse" in these cases as 1 have the feeling, although it may be entirely erroneous, that the majority of these cases occur through reinfection from without and not from auto-infection. On this account I prefer the term " second attack." Doubtless, however, true relapses (cases due to auto-infection) do occur. I shoulả like very much to know if "relapses" are often seen in general practice, as I think that their absence would go a long way to prove that the majority of the cases under discussion are "second attacks" and not relapses in the true sense.

In connexion with this subject I should like very briefly to refer to another class of cases seen occasionally in hospital which differ from the cases above discussed in that they do not desquamate after the first attack. They are what one might call " mild scarlatinal infections" as against scarlet fever. Such cases are admitted with a history of slight sore-throat, transient rash, and slight malaise. On admission to hospital they usually show a normal temperature. They do not desquamate. However, if put in a scarlet fever ward they develop scarlet fever, not immediately after admission, but about three weeks later. The following is a case in point.

CASE 3.-A boy, aged five years, was admitted to the Western Fever Hospital on Sept. 1st, 1902 He bad a slight shivering on August 3lst and slight sore-throat. On Sept. 1st, the day of admission, a rash was said to have been seen. On admission there was no rash; the temperature was $99^{\circ} \mathbf{F}$, the mucous membrane of the fauces was congested, and the tongue was coated and had prominent papillæ. He did not desquamate at all although watched carefully. On the 27th he developed a typical attack of scarlet fever, having a bright rash with a temperature of $103^{\circ}$. He subsequently desquamated freely. In connexion with this case I would specially point out that a sister of this patient was admitted on the same day as he was and that she desquamated typically in the ordinary course. The facts that the boy's sister had scarlet fever and that he did not develop scarlet fever in the ward till three weeks after admission point to his having acquired a certain immunity through the first transient illness and if so it must have been of the nature of scarlet fever.

Fulham, s.W.

\section{A CASE OF AN UNUSUAL NUMBER OF GALL-STONES ; CHOLECYSTOTOMY ; RECOVERY.}

By C. A. S. RIDOUT, M.B., B.S. Lond., L.R.C.P. LoND., M.R.C.S. ENG.,

HOUSE SURGEON TO THE NORTH STAFFOBDSHIRE INFIRMARY, STOKEON-TRENT.

THE following case is one of considerable interest owing to the great number of gall-stones removed at one operation from the gall-bladder. I can find no record of a greater number having been similarly removed and therefore $I$ venture to report the case.

A married woman, aged 43 years, was admitted into the North Staffordshire Infirmary on Dec. 3rd, 1902, under the care of Dr. G. Stokes Hatton, senior surgeon to the infirmary, suffering from abdominal pain and jaundice. As history it was stated that the patient was quite well until five weeks previously when she was seized with a severe attack of pain at the bottom of the back and in the right hypochondrium, accompanied by faintness, sweating, and sickness. The attack lasted acutely for three days, but she had had pain off and on ever since and from Nov. 29th she had been jaundiced-i.e., the jaundice was first noticed four days previous to admission. She stated that she had had many previous attacks of pain of a similar nature and that she had been losing fle:h for the past six months. The patient was the mother of two children and she had had no miscarriages and she menstruated regularly.

On admission, the patient was a thin-faced woman, deeply jaundiced, both skin and conjunctivæ being markedly affected; the tongue was furred and dry and the skin was dry. The pulse was 120 and feeble; the respirations were 28 and quiet; and the temperature was $102^{\circ} \mathrm{F}$. and tending to remit. The urine was acid, with a specific gravity of 1014 and a faint trace of albumin; it contained no sugar and was deeply stained with bile. The bowels were opened by aperients; the frees were clay coloured The lungs were natural, the heart's action was feeble, and 